\title{
Model-based Dynamics and Control: from Cars to Biomechatronics
}

\author{
John McPhee \\ Systems Design Engineering, University of Waterloo \\ Ontario, Canada \\ mcphee@uwaterloo.ca
}

In the Motion Research Group at the University of Waterloo, we investigate the dynamics, model-based control, and design optimization of multibody biomechatronic systems. Deriving the equations for these complex systems is both tedious and error-prone, so we have automated the dynamic modelling process by combining linear graph theory from mathematics with fundamental principles from physics and biology. Our symbolic computer implementation of this approach, now part of MapleSim ${ }^{\mathrm{TM}}$, will generate real-time simulation code and dynamic controllers for systems ranging from biomechatronic devices to autonomous vehicles. In this talk, I will discuss some advantages of a symbolic graph-theoretic approach to systems modelling and control, and highlight these advantages through a large number of real-world applications that include a plug-in Prius for Toyota, a rover for the Canadian Space Agency, a hockey slapshot robot, and a stroke rehabilitation robot that uses an integrated model of the patient's musculoskeletal system and robot to determine optimal control actions. Both modelling and experimental results will be presented to show the real-world performance of the model-based designs and controllers. Future applications to autonomous vehicles and biomechatronic assistive devices will be discussed. 